\title{
EVALUACIÓN ÉTICA DE ATLETAS Y TRIATLETAS ESPAÑOLES SOBRE USO DE LA TECNOLOGIAA VAPORFLY
}

\section{ETHICALEVALUATIONBY SPANISHATHLETES AND TRIATHLETES ON THE USE OF VAPORFLY TECH}

Recibido el 6 de junio de 2021 / Aceptado el 30 de noviembre de 2021 / DOI: 10.24310/riccafd.2021.v10i3.12840 Correspondencia: Jorge de Andrés Sánchez.jorge.deandres@urv.cat

\section{de Andrés-Sánchez J. ${ }^{1 A-F}$ de Torres Burgos F. ${ }^{2 A-F}$}

${ }^{1}$ Social and Business Research Laboratory. Universidad Rovira i Virgili. jorge.deandres@urv.cat. 2Departamento de Economía y Derecho. IES Jaume I de Salou. fdetorresburgos@gmail.com.

Responsabilidades

${ }^{A}$ Diseño de la investigación, ${ }^{B}$ Recolector de datos, ${ }^{C}$ Redactor del trabajo, ${ }^{D}$ Tratamiento estadístico, ${ }^{\mathrm{E}} \mathrm{Apoyo}$ económico, Fldea original y coordinador de toda la investigación.

\section{RESUMEN}

La tecnología Vaporfly (TVF) ha supuesto una disrupción en el fondo en ruta. Entre los problemas éticos que puede provocar cualquier nueva tecnología se encuentran la desnaturalización del deporte, que no sea accesible a todos los competidores y que su uso que entrañe riesgo. A partir de una encuesta a 252 deportistas observamos que la TVF tuvo un juicio ético positivo $(p<0,01)$. No obstante, dicho juicio no es homogéneo en todos los ítems éticos evaluados $(p<0,01)$. Asimismo, el género $(p<0,05)$, el nivel de renta $(p<0,01)$ y practicar triatlón en lugar de atletismo $(p<0,05)$ presentan efectos directos significativos en su percepción moral. También comprobamos que la TVF presenta una valoración más cercana a ser preventiva que generadora de lesiones $(p<0,05)$. El análisis del índice de Haake sobre la evolución de los récords del mundo y de España en diversas distancias de fondo en ruta sugiere que las mejoras de los récords con TVF pueden ser debidos a una lógica evolución del deporte y no a su desnaturalización. 


\section{PALABRAS CLAVE}

escala ética multidimensional, ética en el deporte, psicología del deporte, fondo en ruta, Nike Vaporfly $4 \%$.

\section{ABSTRACT}

Vaporfly technology (VFT) has disrupted road distance running. Among the ethical problems that any new technology can cause we can remark denaturing of sport, that it may be not accessible to all competitors and that its use suppose risk. From a survey of 252 athletes, we observed that the VFT had a positive ethical judgment $(p<0.01)$. However, this judgement is not homogenous in all evaluated items $(p<0.01)$. Likewise, gender $(p<0.05)$, income level $(p<0.01)$ and practicing triathlon instead of athletics $(p<0.05)$ influence the ethical judgment. The VFT presents a perception closer to being preventive than generating injuries $(p$ $<0.05$ ). The analysis of the Haake index on the evolution of world and Spanish records at various distance distances on the road suggests that the improvements in the records with TVF may be due to a logical evolution of the sport and not to its denaturation.

\section{KEY WORDS}

multiple dimensional ethical scale, sport ethics, sport psychology, road distance running, Nike Vaporfly $4 \%$

\section{INTRODUCCIÓN}

En mayo de 2017 culminó el proyecto Breaking2, donde se presentó la tecnología Vaporfly de Nike, concretamente su modelo Vaporfly $4 \%$, que ha supuesto una auténtica disrupción en la concepción del calzado atlético de fondo en ruta y sus consecuencias, han sacudido y están sacudiendo a día de hoy el mundo atlético a todos los niveles: industria, atletas, récords, nuevas reglas de la World Athletics (WA).... En adelante nos referiremos a la tecnología Vaporfly (TVF) sin nombrar explícitamente a Nike ya que desde 2020 diversos competidores como Adidas, Asics, etc. tienen en el mercado modelos con utilizan una tecnología similar.

Hoogkamer et al. (2018), en un estudio que ya habían presentado el 2017, fueron los primeros en medir las mejoras en el rendimiento provocadas por las nuevas zapatillas. La conclusión era que la TVF mejoraba el consumo de oxígeno a un ritmo de carrera determinado, haciéndolo más eficiente. Posteriormente se demostró que dichas mejoras se producen tanto en un plano biomecánico (Hunter et al., 2019; Hoogkamer, et al., 2019), como en el coste energético (Hoogkamer 
et al. 2018; Barnes et al., 2019; Hunter et al., 2019). Quealy y Katz (2018) y Guinness at al. (2020) usando datos de una conocida red social deportiva, observaron que la mejora producida por la TVF es estadísticamente signifi cativa. De los dos elementos clave de la TVF, la placa de fi bra de carbono y la nueva espuma Pebax, fue la placa la que centró toda la atención, ya que fue asimilada a un resorte en la media suela. Dos años antes de la presentación de la TVF, James (2015) había observado que colocando unos muelles en la media suela de una zapatilla experimental, la economía de carrera de los corredores aumentaba signifi cativamente. No obstante, en el caso de la TVF, más importante que el efecto de la placa de carbono, es el asociado a su revolucionaria espuma, que pesa la mitad la de otras marcas y es mucho más efi ciente en el retorno de energía (Hoogkamer et al., 2019; Burns y Tam, 2020).

Hasta enero de 2020, el artículo del reglamento de WA que regulaba el uso de zapatillas estipulaba poco más que "prohibir aquéllas que proporcionaran una ventaja injusta" o que "debían estar razonablemente disponibles en aras a la universalidad del espíritu atlético" sin muchas más aclaraciones. Esta indeterminación normativa suponía que el desarrollo y uso de la TVF se encontrara en un limbo regulatorio (Ross, 2020). Una gran parte de la comunidad atlética exigía una mayor regulación de la TVF (Barfoot, 2020; Burns y Tam, 2020). Incluso las opiniones más puristas califi caron la TVF de doping tecnológico (Woodward, 2020; Ross, 2020) y abogaron por su prohibición (Muñiz-Pardo et al., 2021). El problema, en cualquier caso, no era nuevo. Siempre que surge una tecnología disruptiva en el deporte, se genera un debate sobre si su utilización es moralmente aceptable (Pérez, 2013). Finalmente, a lo largo de 2020 la WA ha ido regulando el uso de la TVF. No obstante, debemos remarcar las tecnologías disruptivas en el deporte no siempre acaban siendo adoptadas. Como ejemplos podemos mencionar los trajes de bañador de poliuretano que la FINA prohibió el año 2010 o, dentro del mismo atletismo, las zapatillas de velocidad de 68 clavos que usó Lee Evans en los trials de Estados Unidos para la clasifi cación a los Juegos Olímpicos de México 68, que fueron prohibidos por la WA (entonces denominada IAAF). Incluso, en ambos casos, las consecuencias finales tampoco son idénticas. Los récords obtenidos con los trajes de poliuretano fueron respetados y su uso es común en otros deportes como el triatlón de media y larga distancia, mientras que las marcas logradas con las zapatillas de 68 clavos fueron suprimidas. Dyer (2015) ofrece una amplia panorámica de controversias éticas producidas por la introducción de tecnologías disruptivas en diferentes deportes.

En este trabajo contrastamos empíricamente a partir de los datos obtenidos con una encuesta sobre deportistas amateurs y analizando la 
evolución de los récords del mundo (RM) y de España (RE) en diferentes distancias de fondo, hasta qué punto podemos sostener que la TVF es poco ética. Entendemos que la elección del segmento de deportistas aficionados es pertinente tanto por el tamaño, que es sustancialmente superior al de profesionales, como por la elevada repercusión económica para las localidades donde se realizan carreras pedestres y triatlones enfocadas a este tipo de deportistas, que ha sido puesta de manifiesto, por ejemplo, en Alguacil et al. (2016). No discutimos si el uso de modelos Vaporfl y de Nike incumplió reglas escritas. No hay duda de que desde un punto de vista formalista su uso era ético, pues la laxa normativa sobre calzado atlético hasta 2020 permitía su empleo y, a partir del 2020, su uso está explícitamente permitido, aunque con limitaciones. En nuestro trabajo nos planteamos su idoneidad desde un punto de vista que Pérez (2013) defi ne como interpretativista, esto es, considerando que la percepción ética "no se reduce a las reglas formales (...) sino que lo que prima sería una serie de principios de innegable vinculatoriedad que permiten concebir al deporte como una actividad significativa y coherente". Aparte de las variables que usualmente inciden sobre la intención de usar nuevas tecnologías de cualquier consumidor como la utilidad percibida, la facilidad de uso o la influencia social, Andrés-Sánchez y Torres-Burgos (2022) muestran que los deportistas amateurs también tienen en cuenta sus percepciones éticas al considerar el uso de zapatillas provistas de TVF. Así, el presente trabajo busca responder a las siguientes preguntas de investigación:

Cuestión 1: Evaluamos si la TVF tiene un juicio ético negativo o positivo por parte de los deportistas

Cuestión 2: El género es una variable relevante para explicar la actitud ante el deporte y la actividad física. Ello nos lleva preguntarnos si el género influye en la valoración de la TVF.

Cuestión 3: Dado que en el atletismo de fondo, la medición de la performance atlética de se realiza, esencialmente, de forma cardinal, mientras que el triatlón se realiza de forma ordinal, ¿Es diferente la valoración ética de las TVF por los atletas y los triatletas?

Cuestión 4: Dado que las zapatillas basadas en la TVF son notablemente más caras que las que no la usan ¿Depende la valoración ética de la TVF de la renta del deportista?

Cuestión 5: ¿Perciben los deportistas un riesgo añadido de lesión debido al uso de la TVF?

Cuestión 6: ¿Depende la percepción del riesgo en la TVF del género, el deporte practicado y el nivel de renta? 
Cuestión 7: La gran cantidad de récords batidos desde la eclosión de la TVF puede llevar a la conclusión de que ésta ha desnaturalizado y roto la integridad del fondo en ruta. No obstante ¿puede sostenerse que la magnitud de las mejoras en los récords es debida de una supuesta desnaturalización del deporte o simplemente fruto de una evolución lógica?

\section{METODOLOGÍA DE ANÁLISIS}

\section{Materiales}

El principal material utilizado en este estudio es una encuesta a 252 atletas y triatletas aficionados de España que fue respondida íntegramente durante marzo de 2021 por medios digitales. La encuesta fue difundida entre clubs de atletismo y triatlón y por redes sociales en grupos de Facebook y WhatsApp compuestos por practicantes de dichos deportes. A dicha encuesta se accedía a través de un link de Google Forms que sólo admitía una respuesta por IP.

La edad media de las personas encuestadas fue 44 años con una desviación estándar de 8,79 años. Un 59\% de los deportistas estaba federado y un $41 \%$ no poseía licencia. La proporción de hombres y mujeres era de $85 / 15$. Asimismo el $67,46 \%$ de respuestas eran corredores y el resto de triatletas. Finalmente, un $42 \%$ de los deportistas admitía una renta mensual superior a $2500 €$.

En la Tabla 1 se puede consultar los ítems empleados y la Tabla 2 la composición de la muestra y los estadísticos descriptivos más relevantes.

Con respecto a la aprobación ética: (1) todos los participantes recibieron información detallada por escrito sobre el estudio y el procedimiento; (2) no se recopilaron datos relacionados directa o indirectamente con la salud de los sujetos y, por lo tanto, la Declaración de Helsinki no se mencionó en general cuando se informó a los sujetos; (3) se aseguró en todo momento el anonimato de los datos recopilados; y (4) no se obtuvo permiso de la aprobación de una junta o comité de ética pues dado el carácter de los datos, no era necesario (5) la cumplimentación voluntaria del cuestionario se tomó como consentimiento para que los datos se utilicen en la investigación. El segundo material utilizado es la evolución de los récords del mundo y de España tanto masculinos como femeninos de maratón, media maratón y $10 \mathrm{~K}$.

Asimismo nuestro análisis también utiliza los registros de los RM y RE masculinos y femeninos en maratón, media maratón y $10 \mathrm{~K}$. 
Tabla 1. Escalas y variables.

\begin{tabular}{|c|c|}
\hline Factor/constructo & Fuente \\
\hline $\begin{array}{l}\text { Juicio ético } \\
\text { Etica1= El uso de zapatillas con placas de carbono me } \\
\text { parece justo (equidad moral) }\end{array}$ & \multirow{6}{*}{$\begin{array}{l}\text { EEM de Shawver anc } \\
\text { Sennetti (2009) }\end{array}$} \\
\hline $\begin{array}{l}\text { Etica2= El uso de zapatillas con placas de carbono me } \\
\text { parece igualitario (equidad moral) }\end{array}$ & \\
\hline $\begin{array}{l}\text { Etica3 = El uso de zapatillas de carbono me parece moral- } \\
\text { mente correcto (equidad moral) }\end{array}$ & \\
\hline $\begin{array}{l}\text { Etica4= El uso de zapatillas con placas de carbono es } \\
\text { aceptable para mis compañeros deportivos más cercanos } \\
\text { (relativismo) }\end{array}$ & \\
\hline $\begin{array}{l}\text { Etica5= El uso de zapatillas con placas de carbono es } \\
\text { aceptable en mi cultura deportiva (relativismo) }\end{array}$ & \\
\hline $\begin{array}{l}\text { Etica6= El uso de zapatillas con placas de carbono es } \\
\text { aceptable para aquéllas personas cuya opinión deportiva } \\
\text { respeto (relativismo) }\end{array}$ & \\
\hline $\begin{array}{l}\text { Riesgo de la TVF } \\
\text { Riesgo=Usar zapatillas con placa de carbono es arriesga- } \\
\text { do (a nivel de lesiones por ejemplo) }\end{array}$ & \\
\hline $\begin{array}{l}\text { Nivel de renta (RENTA) } \\
\text { ¿Con que género te identificas? }\end{array}$ & \\
\hline $\begin{array}{l}\text { Nivel de renta (RENTA) } \\
¿ \text { ¿Es tu renta mensual es al menos } 2500 € ?\end{array}$ & \\
\hline $\begin{array}{l}\text { Deporte de procedencia } \\
\text { ¿Eres atleta/corredor o triatleta? }\end{array}$ & \\
\hline
\end{tabular}

\section{Variables utilizadas}

Las variables relativas a los juicios morales provienen de la escala ética multidimensional (EEM) de Reidenbach y Robin (1990) revisada por Shawver and Sennetti (2009). Se trata de un instrumento de medición de juicios éticos que ha sido muy utilizado en la evaluación del uso de tecnologías disruptivas en ámbitos tan diversos como la enseñanza superior (Junk, 2009); el comercio electrónico (Leonard y Jones, 2017) y, en España, la tecnología ciborg (Pelegrín-Borondo et al., 2020). De las 5 dimensiones contempladas en la escala: equidad moral, relativismo, egoísmo, utilitarismo y contractualismo utilizamos las dos primeras. 
Las preguntas de la encuesta que requieren de una valoración subjetiva han sido contestadas en una escala Likert de 11 niveles que varían desde 0 (valoración totalmente desfavorable) hasta 10 (valoración totalmente de favorable). Así, un 5 implicaría una valoración neutra. En la dimensión equidad moral, la persona encuestada debía valorar si el uso de la TVF es justo, equitativo o igualitario en un sentido amplio. Este aspecto queda reflejado por las 3 primeras preguntas expuestas en la Tabla 1. En la dimensión relativista el/la deportista debía evaluar hasta qué punto su entorno deportivo (compañeros de club, entrenador/a,...) considera admisible o adecuado el uso de la TVF. Esta dimensión queda valorada con las preguntas cuarta, quinta y sexta del cuestionario.

Es un hecho contrastado que el género es un factor determinante en la actitud ante la actividad física y el deporte, tanto en la infancia (Cantero et al., 2019) como en edades adultas. Así, mientras que los deportistas masculinos tienden a entender el deporte desde una perspectiva competitiva, las deportistas se inclinan a objetivos estéticos y de salud (Lores y Murcia, 2008). Por otra parte, la influencia de las redes sociales en contextos deportivo suele ser mayor en las deportistas que en los deportistas (Hazari, 2017; Scott et al., 2021). Entendemos que ambas cuestiones podrían inducir una percepción de TVF dependiente del género.

El deporte de procedencia del encuestado puede tener influencia en la percepción ética de la tecnología Vaporfly. En este sentido, Loland (2009) distingue dos enfoques morales (excluyendo el que él denomina no-enfoque): el enfoque estrecho y el amplio. Según el enfoque estrecho, el objetivo de la regulación de la equipación debe consistir en obtener una medida exacta de los límites del deportista en una determinada disciplina. La tecnología permitida, por tanto, debe estar absolutamente estandarizada y debería mantenerse invariable a lo largo del tiempo. El enfoque amplio es más laxo en cuanto a las necesidades de estandarización. Sería suficiente con que los deportistas tengan las mismas oportunidades de acceso y que cumpla ciertos principios que debemos entender, tal como define Pérez (2013), como enunciados abiertos y difusos que no son exactamente reglas, para considerarla aceptable. Algunos ejemplos serían que sea accesible a todos los contendientes o que no suponga una deshumanización y desnaturalización del deporte.

En el atletismo es muy relevante obtener mediciones precisas (distancia/tiempo). De hecho, para poder participar en determinadas competiciones de cierto relieve (ello abarca desde Juegos Olímpicos hasta competiciones regionales) se requieren unas marcas mínimas. Por otra parte, es común publicar ránquines de atletas en distancias de referencia (desde 60 metros lisos hasta $100 \mathrm{~K}$ en ruta) que abarcan 
diversas generaciones. Así, parece razonable que la percepción que deban tener los atletas sobre los requerimientos de equipamiento deportivo en competición sea asimilable a los de la teoría "estrecha". En cambio, en el triatlón, la medición de la performance deportiva consiste en la posición obtenida dentro de la lista de llegada. La condición para poder participar en un campeonato de mayor relevancia no está basada en un estándar de tiempo utilizado en completar una determinada distancia, sino en haber obtenido clasificaciones notables en otras competiciones de menor relieve. Así, parece razonable que los practicantes de este deporte tengan una concepción más laxa sobre la necesidad de estandarización del material de competición. Por tanto, nuestro análisis incorporará una variable dicotómica (deporte de procedencia) que puede ser atletismo de fondo o triatlón.

Uno de los principios básicos que debe permitir cualquier regulación deportiva es que los contendientes tengan igual facilidad de acceso al equipamiento (Terradillos, 2013; Dyer, 2020). Sin duda, las zapatillas con TVF son sustancialmente más caras que las que no la incorporan (Dyer, 2020). Así, parece razonable suponer que el juicio moral de la TVF también debe estar influido por el nivel de renta del deportista. En nuestro trabajo la variable renta es modelizada como una variable dicotómica que diferencia ingresos menores a $2.500 €$ y superiores a dicha cuantía.

La percepción de la existencia de un problema de accesibilidad a la TVF debido a su precio y su importancia puede ser diferente en función del deporte practicado. Siguiendo a Sailors (2009) "el atletismo ha sido considerado tradicionalmente como el deporte más puro porque únicamente hacen falta dos zapatillas para practicarlo" y, por tanto, el problema de acceso a la TVF puede ser esencial. En cambio, el problema del acceso a determinadas tecnologías debido a su precio es una circunstancia que el triatleta puede tener más asimilado, puesto que las diferencias de precio y relevancia en la performance atlética pueden superiores en los trajes de neopreno para nadar y, sobre todo, en la bicicleta que en las zapatillas para la carrera a pie.

Es un principio ampliamente aceptado que para que una tecnología deportiva sea aceptable es que sea segura (Pérez, 2013; Dyer, 2015). Es cierto que la TVF supone ciertos cambios biomecánicos (Hunter et al., 2019; Hoogkamer, et al., 2019) y éstos pueden generar lesiones (Dyer, 2020). También se ha reportado que una cantidad significativa de atletas populares puede no adaptarse a este tipo de tecnología (Herbert-Losier et al., 2021). No obstante, la sensación de muchos atletas de élite es que la TVF permite sesiones intensas con una mejor recuperación muscular y que su uso genera menores lesiones (Jiménez, 2020). Por otra parte, es ampliamente aceptado que la investigación de nuevas tecnologías en 
el deporte tiene como uno de sus principales objetivos, la prevención de lesiones. Seguramente, dentro del ámbito deportivo, uno de los campos más activos es el de las tecnologías de evaluación y mejora de la biomecánica, que tiene como uno de sus principales objetivos, más allá de acrecentar performance atlética, minimizar el riesgo de lesiones (Mantilla, 2019). Sailors (2009) señala que la prevención de lesiones también es uno de los objetivos primordiales en el desarrollo de la tecnología de calzado deportivo. Este aspecto es valorado a partir del ítem de la Tabla 1 "Usar zapatillas tipo Vaporfly es arriesgado (a nivel de lesiones por ejemplo)", que es evaluado nuevamente en una escala de Likert de 11 niveles.

El supuesto peligro que ha supuesto la TVF para la integridad del deporte ha sido remarcado por Ross (2020) y Woodward (2020). De hecho Muñiz-Pardo et al. (2021) sugieren que pudiera producir mejoras en las performances atléticas similares a las del dopaje sanguíneo. Para evaluar este aspecto ponemos en relación la mejora relativa que suponen los récords batidos con la TVF y las que se registraron antes de su aparición. Bajo esta perspectiva, Dyer (2020) realiza un análisis del progreso que han ido experimentando los RM masculinos y femeninos de maratón y media maratón. En su opinión la mejora de marcas no sugiere un despegue abrupto de los tiempos debido al uso de la TVF, sino que son compatibles con una lógica evolución de las disciplinas evaluadas.

\section{Metodología cuantitativa}

Como paso previo al análisis estadístico que permita dar respuesta a las preguntas de investigación formuladas, medimos la fiabilidad interna de la escala ética expuesta en la Tabla 1. Para ello calculamos su alfa de Cronbach, su fiabilidad compuesta y la varianza media extraída. Complementamos dichas medidas con un análisis factorial confirmatorio.

La primera pregunta de esta investigación ¿Tiene realmente la TVF un juicio ético negativo? fue contestada contrastando la hipótesis nula de que la valoración media/mediana de cada uno de los 6 ítems éticos es 5 (ni positivo ni negativo) con las pruebas $t$ de Student y de rangos por signos de Wilcoxon. Asimismo contrastamos si los seis ítems son evaluados de forma homogénea con ANOVA.

Las tres siguientes preguntas que realizamos ¿Es el género del/de la deportista relevante en el juicio ético sobre la TVF? ¿Es el deporte de procedencia atletismo/triatlón relevante en el juicio ético sobre la TVF? y ¿Es el nivel de renta del deportista relevante en su juicio ético sobre la TVF? fueron testadas realizando una análisis MANOVA sobre los ítems éticos, en los que las variables discriminantes fueron dichos factores explicativos. 
La quinta pregunta ¿Percibe el atleta un nivel de riesgo añadido por el uso de la TVF? fue contestada testando la hipótesis nula consistente en que la valoración media/mediana del ítem relativo al riesgo era 5 (ni acuerdo ni desacuerdo en el enunciado). Asimismo, la sexta cuestión, en la que sopesamos la influencia del género, renta y deporte practicado sobre la percepción de riesgo, es evaluada a partir de los resultados de un análisis de la varianza con tres factores.

La séptima pregunta de investigación, ¿puede sostenerse que la magnitud de las mejoras en los récords es fruto de una supuesta desnaturalización del deporte o simplemente fruto de su evolución?, es contestada complementando los resultados de Dyer (2020) con los que nosotros obtenemos respecto a la evolución del RM de $10 \mathrm{~K}$ masculino y femenino y el RE de maratón, media maratón y $10 \mathrm{~K}$ masculinos y femeninos. Como Dyer (2020), utilizamos el índice de mejora de Haake (IMH), que se define como:

$$
I M H=\left[\left(\frac{t_{a}}{t_{p}}\right)^{2}-1\right] \cdot 100
$$

siendo $t_{a}$ la marca registrada en el récord anterior y $t_{p}$ la marca del récord posterior.

\section{RESULTADOS}

La Tabla 2 muestra que la escala ética utilizada presenta consistencia interna. Los valores del alfa de Cronbach y la fiabilidad compuesta son $>0,7$, la varianza media extraída es $>0,5$ y la extracción factorial de todos los ítems rebasa sobradamente 0,7 .

Respecto a la primera pregunta de la investigación, en la Tabla 2 puede observarse que las medidas de tendencia central (media y mediana) de los ítems de valoración ética fueron notablemente superiores a 5 . En la Tabla 3 dichos promedios vienen diferenciados por género, deporte y nivel de renta, observándose que en todos los casos las evaluaciones son también postivas. En la Tabla 2 se observa que la prueba t de Student (sobre media) y la de Wilcoxon (para la mediana) rechazaron la neutralidad de la valoración en todas las preguntas $(p<0,01)$. En conclusión, en nuestra muestra se rechazó que el uso de la TVF presente problemas desde el punto de vista de la ética deportiva. De hecho la tendencia central señaló una valoración positiva significativa. No obstante, puede observarse que el segundo ítem ético, relativo a la percepción la influencia de la TVF en la igualdad competitiva y perteneciente al concepto de equidad moral, presenta una valoración promedio de 5,67; sustancialmente inferior al resto, que son evaluados 
por encima del 7. Los resultados del ANOVA, expuestos en la Tabla 4 , sugieren que existe homogeneidad en las respuestas de todos los ítems con excepción del segundo. Así, cuando consideramos los 6 ítems rechazamos la homogeneidad de medias $(F=13,55, p<0,01)$. En cambio, cuando eliminamos del análisis ANOVA el segundo ítem, el resultado apunta a la existencia de homogeneidad en las respuestas sobre el resto de ítems éticos $(F=1,03, p=0,389)$.

\section{Tabla 2. Estadísticas descriptivas de la muestra y resultados de la validación de la escala ética}

\begin{tabular}{llllllllll}
\hline \multicolumn{1}{c}{ Estadística descriptivas } & \multicolumn{1}{c}{ Validación de escalas } \\
\hline item & media & mediana & DE & Q1 & Q3 & $\alpha-C$ & FC & VME & EF \\
\hline Etica1 & $7.13^{* * *}$ & $8.00^{* * *}$ & 2,890 & 5,00 & 10,00 & 0,958 & 0,967 & 0,832 & 0,934 \\
Etica2 & $5.67^{* * *}$ & $6.00^{* * *}$ & 3,235 & 3,00 & 9,00 & & & & 0,835 \\
Etica3 & $7.00^{* * *}$ & $7.00^{* * *}$ & 3,010 & 5,00 & 10,00 & & & & 0,948 \\
Etica4 & $7.33^{* * *}$ & $8.00^{* * *}$ & 2,845 & 6,00 & 10,00 & & & & 0,938 \\
Etica5 & $7.33^{* * *}$ & $8.00^{* * *}$ & 2,928 & 6,00 & 10,00 & & & 0,945 \\
Etica6 & $7.46^{* * *}$ & $8.00^{* * *}$ & 2,583 & 6,00 & 10,00 & & & 0,863 \\
\hline Riesgo & $4.54^{* * *}$ & $4.50^{* *}$ & 2,727 & 3,00 & 6,00 & & & \\
\hline Deportistas con renta mensual $\geq 2500 €=42 \%$ \\
Proporción atletas/triatletas=67,46\% vs $32.54 \%$. \\
Composición por sexo: $85 \%$ hombres y $15 \%$ mujeres \\
Composición por edades: $15 \%$ menores de 35 años; $42 \%$ entre 35 y 45 años; $36 \%$ entre 45 y 55 años; $6 \%$ \\
entre 55 y 65 años y el $1 \%$ mayores de 65 años.
\end{tabular}

Notas: DE es desviación estándar, Q1 y Q3 son el primer y tercer cuartil. Asimismo a-C es el alfa de Cronbach, FC el índice de fiabilidad compuesta, VME la varianza media extraída y EF la extracción factorial (rotación varimax). Asimismo, “**” y “***” denota rechazo de que la tendencia central toma valor 5 con niveles de significación del $10 \%, 5 \%$ y $1 \%$, respectivamente.

Tabla 3. Media y desviación estándar de las preguntas discriminadas por género, deporte y renta.

\begin{tabular}{|l|l|l|l|l|l|l|l|l|l|l|}
\hline \multirow{2}{*}{ Item } & Género & Media & DE & \multicolumn{1}{|c|}{ Deporte } & \multicolumn{1}{l|}{ Media } & DE & Renta & Media & DE \\
\hline \multirow{2}{*}{ Ética1 } & femenino & 7,56 & 2,14 & atleta & 7,01 & 3,03 & $<2.500 €$ & 7,19 & 2,88 \\
\cline { 2 - 11 } & masculino & 7,15 & 2,91 & triatleta & 7,66 & 2,21 & $>=2.500 €$ & 7,22 & 2,73 \\
\hline \multirow{2}{*}{ Ética2 } & femenino & 6,28 & 2,50 & atleta & 5,51 & 3,20 & $<2.500 €$ & 5,86 & 3,24 \\
\cline { 2 - 11 } & masculino & 5,67 & 3,30 & triatleta & 6,34 & 3,14 & $>=2.500 €$ & 5,59 & 3,13 \\
\hline \multirow{2}{*}{ Ética3 } & femenino & 7,33 & 2,29 & atleta & 6,84 & 3,10 & $<2.500 €$ & 6,86 & 3,05 \\
\cline { 2 - 11 } & masculino & 7,05 & 3,04 & triatleta & 7,68 & 2,44 & $>=2.500 €$ & 7,45 & 2,76 \\
\hline
\end{tabular}




\begin{tabular}{|c|c|c|c|c|c|c|c|c|c|}
\hline Item & Género & Media & $\mathrm{DE}$ & Deporte & Media & DE & Renta & Media & DE \\
\hline \multirow[t]{2}{*}{ Ética4 } & femenino & 8,22 & 1,29 & atleta & 7,31 & 2,83 & $<2.500 €$ & 6,99 & 3,07 \\
\hline & masculino & 7,28 & 2,92 & triatleta & 7,63 & 2,61 & $>=2.500 €$ & 8,08 & 2,05 \\
\hline \multirow[t]{2}{*}{ Ética5 } & femenino & 8,11 & 1,43 & atleta & 7,28 & 2,93 & $<2.500 €$ & 7,12 & 3,06 \\
\hline & masculino & 7,30 & 3,01 & triatleta & 7,74 & 2,66 & $>=2.500 €$ & 7,90 & 2,42 \\
\hline \multirow[t]{2}{*}{ Ética6 } & femenino & 8,50 & 1,44 & atleta & 7,24 & 2,70 & $<2.500 €$ & 7,32 & 2,88 \\
\hline & masculino & 7,37 & 2,69 & triatleta & 8,21 & 2,13 & $>=2.500 €$ & 7,86 & 1,97 \\
\hline \multirow{2}{*}{$\begin{array}{l}\text { Ries- } \\
\text { go }\end{array}$} & femenino & 4,17 & 2,42 & atleta & 4,53 & 2,78 & $<2.500 €$ & 4,32 & 2,87 \\
\hline & masculino & 4,55 & 2,77 & triatleta & 4,42 & 2,60 & $>=2.500 €$ & 4,78 & 2,47 \\
\hline
\end{tabular}

Nota: DE es desviación estándar

Tabla 4. Resultados del análisis ANOVA para la homogeneidad de respuestas los ítems éticos

\begin{tabular}{|l|l|l|}
\hline Hipótesis nula & Estadístico F & p-valor \\
\hline Ética1=Ética2=Ética3=Ética4=Ética5=Ética6 & 13,55 & $<0,01$ \\
\hline Ética1= Ética3=Ética4=Ética5=Ética6 & 1,03 & 0,389 \\
\hline
\end{tabular}

Los resultados de la Tabla 3 sugieren que las mujeres, los triatletas y las personas con una renta mensual mayor de $2.500 €$ tienen una mejor percepción ética de la TVF. La significación de este resultado se contrasta mediante un análisis MANOVA, cuyos resultados son expuestos en la Tabla 5. Observamos que el efecto directo de los tres factores evaluados, género $(p<0,05)$, nivel de renta $(p<0,01)$ y deporte de procedencia $(p<0,05)$ tiene una influencia significativa en el juicio efectuado sobre la TVF. La interacción entre los tres factores también es relevante en la formación del juicio ético $(p<0,01)$. En cambio, la interacción por pares de los factores evaluados no tiene impacto significativo en el juicio del deportista.

Tabla 5. Resultados del análisis MANOVA de las preguntas relativas a la ética

\begin{tabular}{|l|l|l|l|l|l|}
\hline \multirow{2}{*}{ Efecto } & \multicolumn{2}{|c}{ Estadístico } & Valor & Efecto & \multicolumn{2}{l|}{ Estadístico } & \multicolumn{1}{l|}{ Valor } \\
\hline Constante & Traza de Pillai & $0,817^{* * *}$ & Género-deporte & Traza de Pillai & 0,024 \\
\hline & Lambda de Wilks & $0,183^{* * *}$ & & Lambda de Wilks & 0,976 \\
\hline & Traza de Hotelling & $4,475^{* * *}$ & & Traza de Hotelling & 0,025 \\
\hline & Raiz de Roy & $4,475^{* * *}$ & & Raiz de Roy & 0,025 \\
\hline
\end{tabular}




\begin{tabular}{|c|c|c|c|c|c|}
\hline Efecto & Estadístico & Valor & Efecto & Estadístico & Valor \\
\hline \multirow[t]{4}{*}{ Género } & Traza de Pillai & $0,060 * *$ & Género-renta & Traza de Pillai & 0,042 \\
\hline & Lambda de Wilks & $0,940 * *$ & & Lambda de Wilks & 0,958 \\
\hline & Traza de Hotelling & $0,064^{* *}$ & & Traza de Hotelling & 0,044 \\
\hline & Raiz de Roy & $0,064^{* *}$ & & Raiz de Roy & 0,044 \\
\hline \multirow[t]{4}{*}{ Género } & Traza de Pillai & $0,062 * *$ & Deporte-renta & Traza de Pillai & 0,040 \\
\hline & Lambda de Wilks & $0,938^{* *}$ & & Lambda de Wilks & 0,960 \\
\hline & Traza de Hotelling & $0,066^{* *}$ & & Traza de Hotelling & 0,042 \\
\hline & Raiz de Roy & $0,066 * *$ & & Raiz de Roy & 0,042 \\
\hline \multirow[t]{4}{*}{ Renta } & Traza de Pillai & $0,087^{* * *}$ & $\begin{array}{l}\text { Género-deporte- } \\
\text { renta }\end{array}$ & Traza de Pillai & $0,094 * * *$ \\
\hline & Lambda de Wilks & $0,913^{* * *}$ & & Lambda de Wilks & $0,906 * * *$ \\
\hline & Traza de Hotelling & $0,096 * * *$ & & Traza de Hotelling & $0,104 * * *$ \\
\hline & Raiz de Roy & $0,096 * * *$ & & Raiz de Roy & $0,104 * * *$ \\
\hline
\end{tabular}

Con “*”, “**” y “***” denotamos rechazo de la hipótesis nula de que el efecto analizado no tiene incidencia en el conjunto de juicios éticos,

La Tabla 2 muestra la percepción que tienen los atletas del riesgo de lesión que puede producir la TVF, y presenta una tendencia central ligeramente inferior de 5 . Dicha diferencia respecto a 5 se mostró estadísticamente significativa ( $p<0,01$ en el test de media y $p<0,05$ en el de mediana). Así no podemos sostener que los deportistas de nuestra muestra percibieran que las zapatillas generaran un riesgo adicional de lesión respecto a otras zapatillas, sino al contrario.

La Tabla 3 muestra que, en promedio, las mujeres, los triatletas y los atletas con niveles de renta mensual por debajo de $2.500 €$ perciben menos riesgo que los hombres, los atletas y las personas con renta mensual superior a $2500 €$. No obstante, la evaluación del ítem está en todos los casos por debajo de 5. El análisis ANOVA de la Tabla 6 sugiere que no existe un efecto directo significativo de los factores considerados en la percepción de la TVF como generadora de lesiones. No obstante, la interacción de los 3 factores sí que puede influir significativamente en la percepción del riesgo $(\mathrm{p}<0,01)$.

Tabla 6. Análisis ANOVA de la incidencia de los factores ensayados en la percepción del riesgo

\begin{tabular}{|l|l|l|}
\hline Efecto & Estadístico $F$ & p-valor \\
\hline Constante & 273,443 & 0,000 \\
\hline Género & 0,130 & 0,719 \\
\hline
\end{tabular}




\begin{tabular}{|l|l|l|}
\hline Efecto & Estadístico $F$ & $p$-valor \\
\hline Deporte & 0,022 & 0,883 \\
\hline Renta & 0,067 & 0,795 \\
\hline Género-deporte & 0,025 & 0,874 \\
\hline Género-renta & 1,381 & 0,241 \\
\hline Deporte-renta & 1,929 & 0,166 \\
\hline Género-deporte-renta & 6,649 & 0,011 \\
\hline
\end{tabular}

El análisis del IMH (Tabla 7) sugirió que las mejoras producidas en los $\mathrm{RM}$ y $\mathrm{RE}$ en las distancias más relevantes del fondo en ruta (maratón, media maratón y 10K) calzando TVF no supusieron un ningún caso un incremento abrupto respecto a los que se había movido antes de la irrupción de la TVF. De hecho el RE de media maratón masculina y el de maratón femenino no han sido batidos desde la aparición de la TVF.

La Tabla 8 sintetiza las preguntas que hemos efectuado en nuestra investigación y la respuesta que damos a la luz de los análisis cuantitativos efectuados.

Tabla 7. Índices de mejora de Haake para los récords del mundo y de España

\begin{tabular}{|l|l|l|l|}
\hline Prueba & Ámbito & IMH & Orden \\
\hline Maratón & RM masculino & $2.1(7.2)$ & 14 de 38 \\
& RM femenino & $2(9.2)$ & 21 de 30 \\
& RE masculino & $0.45(8.88)$ & 24 de 32 \\
& RE femenino & no batido & \\
\hline Media Maratón & RM masculino & $0.98(7.5)$ & 11 de 32 \\
& RM femenino & $0.7(6.2)$ & 15 de 27 \\
& RE masculino & no batido & \\
\hline \multirow{2}{*}{$10 \mathrm{~K}$} & RE femenino & $0.38(3.85)$ & 3 de 3 \\
\hline & RM masculino & $1.78(3.83)$ & 9 de 20 \\
& RM femenino & $4.3(7.93)$ & 5 de 16 \\
& RE masculino & $0.84(0.95)$ & 2 de 5 \\
& RE femenino & $0.84(2.74)$ & 3 de 3 \\
\hline
\end{tabular}

Nota: En la columna IMH sin paréntesis viene dado el IMH máximo desde 2016, mientras que en paréntesis viene ese mismo valor para el conjunto de mejoras analizado 
Tabla 8. Síntesis de los resultados obtenidos en nuestro trabajo

\begin{tabular}{|l|l|}
\hline Pregunta de investigación & Conclusión \\
\hline $\begin{array}{l}\text { ¿Tiene realmente la TVF un juicio ético negativo? } \\
\text { ¿Depende la valoración ética de las TVF del gé- }\end{array}$ & $\begin{array}{l}\text { No, al menos en nuestra encuesta. No obstante } \\
\text { ítem relativo a la influencia sobre la igualdad en- } \\
\text { tre competidores presenta una valoración signifi- } \\
\text { cativamente inferior al resto. }\end{array}$ \\
$\begin{array}{l}\text { nero? } \\
\text { ¿Es diferente la valoración ética de las TVF por los } \\
\text { atletas y los triatletas? }\end{array}$ & $\begin{array}{l}\text { que los hombres } \\
\text { ¿i, los triatletas tienen una mejor percepción ética } \\
\text { de la TVF }\end{array}$ \\
$\begin{array}{l}\text { ¿Depende la valoración ética de la TVF de la renta } \\
\text { del deportista? }\end{array}$ & $\begin{array}{l}\text { Sí, un mayor nivel de renta está asociado a una } \\
\text { mejor percepción de la TVF }\end{array}$ \\
$\begin{array}{l}\text { ¿Perciben los deportistas un riesgo añadido por el } \\
\text { uso de la TVF? }\end{array}$ & $\begin{array}{l}\text { No. En el peor de los casos su valoración es neu- } \\
\text { tra. }\end{array}$ \\
$\begin{array}{l}\text { ¿Influyen el género, el deporte practicado y el niv- } \\
\text { el de renta en la percepción de riesgo de la TVF? }\end{array}$ & $\begin{array}{l}\text { No se aprecian efectos directos significativos. No } \\
\text { obstante, la interacción de las tres variables pre- } \\
\text { senta un impacto en dicha percepción estadística- } \\
\text { mente significativa }\end{array}$ \\
$\begin{array}{l}\text { ¿Puede sostenerse que la magnitud de las mejo- } \\
\text { ras en los récords es fruto de una supuesta des- } \\
\text { naturalización del deporte o simplemente fruto } \\
\text { de su evolución? }\end{array}$ & $\begin{array}{l}\text { El análisis del índice de Haake sugiere que es fruto } \\
\text { de una evolución razonable de la disciplina. }\end{array}$ \\
\hline
\end{tabular}

\section{DISCUSIÓN Y CONCLUSIONES}

Existen diversos estudios que reportan que la TVF de Nike, presentada el año 2017 en el evento Breaking2, proporciona ventaja sobre el resto de modelos de zapatilla de competición existentes entonces. Esta cuestión ha generado un enconado debate entre los autores que abogan por su prohibición y los entienden que es un avance tecnológico que puede ser asumido si es regulado adecuadamente. La prolija normativa sobre las zapatillas de atletismo desarrollada el año 2020 por la World Athletics iría en el sentido de la segunda corriente de opinión (TorresBurgos y Andrés-Sánchez, 2021). Si bien, el análisis ético de la TVF ha sido tratado ampliamente en diversos trabajos con una metodología discursiva, los estudios empíricos al respecto son escasos. Este hecho motiva el presente artículo, que evalúa empíricamente los supuestos problemas morales asociados a la irrupción de los modelos de Nike Vaporfly en el fondo en ruta. El principal instrumento empleado es una encuesta efectuada a 252 atletas amateurs. Concretamente, valoramos si los encuestados entienden que el uso de la TVF rompe principios éticos básicos como justicia, igualdad o equidad; si supone un riesgo para los atletas y puede considerarse que rompe la integridad del deporte dado 
el supuesto cambio abrupto de las performances atléticas observadas desde el año 2017.

La encuesta analizada revela una opinión positiva y significativamente por encima de 5 en una escala de 0-10 en todos los ítems éticos. No obstante, hemos observado que la evaluación del segundo ítem de la EEM, "el uso de zapatillas con placas de carbono me parece igualitario", inscrito dentro del concepto de equidad moral, presenta un evaluación significativamente inferior al del resto, para los que, en cambio, sí que apreciamos homogeneidad en su valoración. Es decir, dentro de la evaluación positiva que presenta la TVF, el aspecto de igualdad de oportunidades entre contendientes es el que presenta peor valoración. Asimismo, aunque la percepción ética positiva se da en ambos géneros, tanto en atletas como de triatletas y en todos los niveles de renta, también es cierto que dichas variables generan un juicio ético diferente.

Las mujeres evalúan mejor los hombres la TVF. A priori puede parecer contradictorio con el hecho de que la TVF mejora la performance en las carreras y las mujeres tienden a practicar deporte con objetivos relacionados con la salud y el bienestar mientras que los hombres suelen enfocarse a aspectos más competitivos. No obstante, también es probable que esta misma circunstancia induzca a los atletas masculinos una valoración de la TVF más extrema (positiva o negativa) en función de su capacidad para acceder a ella y sus códigos éticos individuales. Esta intuición se vería confirmada por la mayor dispersión en las contestaciones de los hombres. Por otra parte, las mujeres son más sensibles a la influencia de las redes sociales y medios de comunicación, que está relacionada con los ítems relativos a la dimensión relativista de la EEM (cuarto, quinto y sexto). Ello podría predisponerlas a una percepción más positiva. De hecho, es en estos ítems donde la diferencia valorativa entre mujeres y hombres es mayor. Debe remarcarse que la TVF ha tenido una difusión en los medios de comunicación y redes sociales muy superior a la de cualquier otro modelo nuevo de zapatillas, tanto por la gran cantidad de récords batidos con ella, como por la espectacularidad de eventos como el Breaking2 o el Ineos1.59 que han servido para presentarla.

Los triatletas valoran mejor la TVF que los atletas. Este hecho pudiera imputarse a la propia naturaleza del triatlón, que entiende la necesidad de estandarización del equipamiento deportivo en el sentido amplio de Loland (2009). Asimismo, los triatletas están más habituados desequilibrios que produce la disparidad de precios en el equipamiento para la natación y el ciclismo.

Las rentas altas valoran mejor la TVF. Ello podría ser debido a que perciben la tecnología como más accesible. La significatividad 
estadística de esta variable estaría de acuerdo con que el aspecto peor valorado en la irrupción de la TVF es el ítem relacionado con la igualdad entre competidores, ya que el precio de las zapatillas con TVF es lo suficientemente elevado como para constituirse como una barrera para su adquisición.

No observamos una percepción negativa de la TVF respecto a que pueda provocar más lesiones. Al contrario, la evaluación promedio sugiere que se percibe como ligeramente protectora. Este hallazgo sería coherente con las sensaciones manifestadas por diversos atletas de élite ya comentadas en el apartado 2.2. y al hecho de que los avances tecnológicos en el deporte suelen tener, entre otros objetivos, la prevención de lesiones. A pesar de que ser mujer, practicante de triatlón y tener una renta inferior a $2.500 €$ predispone a percibir la TVF como preventiva de lesiones, dichas variables no tienen un impacto directo significativo sobre la percepción de riesgo.

Los valores obtenidos para el índice de Haake nos llevan a obtener la misma conclusión que Dyer (2020): las mejoras registradas en las performances no sugieren cambios bruscos en la evolución natural de las disciplinas de fondo evaluadas (10K, media maratón y maratón) en los ámbitos analizados (récords del mundo y de España).

El estudio presenta diversas limitaciones que pueden incentivar el desarrollo de futuras investigaciones. El ratio hombres/mujeres, $85 / 15$, está cerca de las proporciones de las proporciones registradas habitualmente en los eventos de fondo en ruta y triatlón de España. En la maratón de Barcelona de 2019 la participación femenina fue del 18\%. No obstante, entendemos que hubiera sido preferible una proporción más paritaria. Este estudio se ha realizado una vez la WA reguló las zapatillas de fondo en ruta. En un contexto que no tiene porqué ser el atletismo, sería interesante desarrollar un estudio similar para una tecnología que pueda proporcionar mejoras en la performance atlética antes de que sea regulada para, posteriormente comparar los resultados con los se obtendrían después de que ésta ya haya sido objeto normativo.

Asimismo, entendemos que podíamos haber ampliado el análisis del índice de Haake en otras disciplinas de ruta (como por ejemplo la milla o los 100k), a otros ámbitos geográficos o a pruebas de pista.

\section{Fondos}

Los autores declaran no recibir financiación.

\section{Agradecimientos}

Los autores agradecen los útiles comentarios y la discusión sobre el tema con todos sus compañeros atléticos (grupos de entreno, clubs, etc.) y de dos evaluadores anónimos. 


\section{Conflictos de interés}

Los autores declaran no tener ningún conflicto de interés.

\section{BIBLIOGRAFÍA}

1. Alguacil, M., Pérez Campos, C., Aguado Berenguer, S., \& Boquera, J. ¿ La celebración de un evento promociona la ciudad como destino turístico entre los deportistas?. Sportk, 2016, 5(1),Supl. https://doi. org/10.6018/254131

2. Andrés-Sánchez, J.; Torres-Burgos, F. Why disruptive sport competition technologies are used by amateur athletes? An analysis of Vaporfly shoes with an UTAUT model, 2022, Journal of Sport of Health Research, Aceptado para su publicación.

3. Barfoot, A. Those superfast Nike Shoes are creating a problem. New York Times, 18/10/2019, [consultado en mayo de 2021], https://www. nytimes.com/2019/10/18/sports/marathon-running-nike-vaporfly-shoes. html?searchResultPosition=12.

4. Barnes, K. R., \& Kilding, A. E. A randomized crossover study investigating the running economy of highly-trained male and female distance runners in marathon racing shoes versus track spikes. Sports Medicine, 2019; 49(2), 331-342, https://doi.org/10,1007/s40279-018-1012-3.

5. Burns, G. T., \& Tam, N.. Is it the shoes? A simple proposal for regulating footwear in road running. British Journal of Sports Medicine, 2020; 54, 4, 1-2. http://dx.doi.org/10.1136/bjsports-2018-100480.

6. Cantero, P., Mayor, A., Toja, B. y González Valeiro, M. Fomento de estilos de vida activos en la escuela: práctica de actividad física, edad y género. Sportis. Scientific Journal of School Sport, Physical Education and Psychomotricity, 2019, 5 (1), 53-69. DOI:https://doi.org/10.17979/sportis.2019.5.1.3680.

7. Dyer, B. A pragmatic approach to resolving technological unfairness: The case of Nike's Vaporfly and Alphafly running footwear. Sports Medicine-Open, 2020; 6, 1-10, https://doi.org/10,1186/s40798-020-00250-1.

8. Dyer, B. The controversy of sports technology: a systematic review. SpringerPlus, 2015; 4(1), 1-12. DOI: 10.1186/s40064-015-1331-x

9. Guinness, J., Bhattacharya, D., Chen, J., Chen, M., \& Loh, A. An Observational Study of the Effect of Nike Vaporfly Shoes on Marathon Performance. arXiv preprint arXiv:2002, 2020; 06105.

10. Hazari, S. Investigating social media consumption, sports enthusiasm, and gender on sponsorship outcomes in the context of Rio Olympics, International Journal of Sports Marketing and Sponsorship, 2018, 19 (4), 396414. https://doi.org/10.1108/IJSMS-01-2017-0007 
11. Hébert-Losier, K., Finlayson, S. J., Driller, M. W., Dubois, B., Esculier, J. F., \& Beaven, C. M. Metabolic and performance responses of male runners wearing 3 types of footwear: Nike Vaporfly 4\%, Saucony Endorphin racing flats, and their own shoes. Journal of Sport and Health Science. 2021; https://doi.org/10,1016/j.jshs.2020.11.012

12. Hoogkamer, W., Kipp, S., \& Kram, R. The biomechanics of competitive male runners in three marathon racing shoes: a randomized crossover study. Sports Medicine, 2019; 49(1), 133-143, https://doi.org/10,1007/ s40279-018-1024-z

13. Hoogkamer, W., Kipp, S., Frank, J. H., Farina, E. M., Luo, G., \& Kram, R. A comparison of the energetic cost of running in marathon racing shoes. Sports Medicine, 2018; 48(4), 1009-1019. https:// doi.org/10.1007/s40279017-0811-2.

14. Hunter, I., McLeod, A., Valentine, D., Low, T., Ward, J., \& Hager, R. Running economy, mechanics, and marathon racing shoes. Journal of Sports Sciences, 2019; 37(20), 2367-2373, https://doi.org/10,1080/02640 414,2019,1633837.

15. Jiménez, C. Los atletas españoles nos cuentan qué opinan sobre las polémicas zapatillas Vaporfly de Nike. Runnersworld Magazine. [consultado en mayo de 2021], https://www.runnersworld.com/es/zapatillas-corrermaterial-deportivo/a30671771/atletas-opinion-zapatillas-nike-vaporfly/

16. Jung, I. Ethical judgments and behaviors: Applying a multidimensional ethics scale to measuring ICT ethics of college students. Computers \& Education, 2009; 53(3), 940-949. https://doi.org/10.1080/10508422.2012.67 2907.

17. Leonard, L. N. \& Jones, K. (2017). Ethical awareness of seller's behavior in consumer-to-consumer electronic commerce: Applying the multidimensional ethics scale. Journal of Internet Commerce, 16(2), 202-218.

18. Loland, S. The ethics of performance-enhancing technology in sport. Journal of the Philosophy of Sport, 2009; 36(2), 152-161. https://doi.org/ $10.1080 / 00948705.2009 .9714754$

19. Lores, A. P., \& Murcia, J. A. M. Actitud de los universitarios ante la práctica físico-deportiva: diferencias por géneros. Revista de psicología del deporte, 2008, 17(1), 7-23. Disponible en: https: //www.redalyc.org/ articulo.oa?id=235119246001

20. Mantilla, J. I. A. Herramientas tecnológicas para el estudio e intervención de la biomecanica en el deporte de alto rendimiento: una mirada desde fisioterapia. Revista Iberoamericana de Ciencias de la Actividad Física y el Deporte, 2019, 8(3), 67-78. https://doi.org/10.24310/riccafd.2019. v8i3.7491

21. McGuire J. They're not just race shoes. Nike's VP of footwear innovation says of the Alphaflys. [consultado en mayo de 2021], https://www. runnersworld.com/uk/gear/shoes/a31240752/what-nike-say-alphafly/. 
22. Muñiz-Pardos, B., Sutehall, S., Angeloudis, K., Guppy, F. M., Bosch, A., \& Pitsiladis, Y. Recent Improvements in Marathon Run Times Are Likely Technological, Not Physiological. Sports Medicine, 2021; 51, 371-378. https: / /doi.org/10,1007/s40279-020-01420-7

23. Pelegrín-Borondo, J., Arias-Oliva, M., Murata, K., \& Souto-Romero, $M$. Does ethical judgment determine the decision to become a cyborg?. Journal of Business Ethics, 2020; 161(1), 5-17, https://doi.org/10,1007/ s10551-018-3970-7

24. Pérez Triviño, J. L.. La filosofía del deporte: Un panorama general. FairPlay, Revista de Filosofia, Ética y Derecho del Deporte, 2013; (1), 3-26.

25. Quealy, K.; Katz, K. Nike Says Its $\$ 250$ Running Shoes Will Make You Run Much Faster. What if That's Actually True? The New York Times. 8th July, 2018. 2018; https://www.nytimes.com/interactive/2018/07/18/upshot/nike-vaporfly-shoe-strava.html

26. Reidenbach, R. E., \& Robin, D. P. Toward the development of a multidimensional scale for improving evaluations of business ethics. Journal of Business Ethics, 1990; 9(8), 639-653. https://doi.org/10.1007/ BF00383391.

27. Riess, K.J.. Mechanical spring technology improves running economy in endurance runners. Journal of Human Sport and Exercise, 2015, (9) 4, 782-789, doi:https://doi.org/10.14198/jhse.2014.94.05.

28. Ross, T. Running shoe tech: The Emperor's clothes, and the issues for the integrity of running. The Science of Sport, February 2020. 2020; https: / /sportsscientists.com/2020/02/running-shoe-tech-the-emperorsclothes-and-the-issues-for-the-integrity-of-running/

29. Sailors, P. R. More than a pair of shoes: Running and technology. Journal of the Philosophy of Sport, 2009; 36(2), 207-216. https://doi.org/10.1 080/00948705.2009.9714757

30. Scott, C. L., Haycraft, E., \& Plateau, C. R. The influence of social networks within sports teams on athletes' eating and exercise psychopathology: A longitudinal study. Psychology of Sport and Exercise, 2021, 52, 101786, https://doi.org/10.1016/j.psychsport.2020.101786

31. Shawver, T. J., \& Sennetti, J. T. Measuring ethical sensitivity and evaluation. Journal of Business Ethics, 2009; 88(4), 663-678.

32. Terradillos, J.i Qué Fair Play?i Qué deporte?. FairPlay, Revista de Filosofia, Ética y Derecho del Deporte, 2013; 1, 44-56.

33. Torres Burgos, F., Andrés-Sánchez, J. (2021). Tecnología Vaporfly en zapatillas de fondo en ruta: algunas consideraciones éticas sobre uso y regulación. FairPlay, Revista de Filosofia, Ética y Derecho del Deporte, (20), 68-89, https://raco.cat/index.php/FairPlay/article/view/393497 
34. Woodward, A. Nike's controversial Vaporfly shoes are helping runners set new records, but some think it's 'technology doping.' Here's how they work. Insider, [consultado en mayo de 2021], https: / /www.businessinsider.com/why-nike-vaporfly-shoes-make-runners-faster-2019-11. 